\title{
Long term effect of alpha interferon in children with chronic hepatitis B
}

F Bortolotti, P Jara, C Barbera, G V Gregorio, A Vegnente, L Zancan, L Hierro, C Crivellaro, G Mieli Vergani, R Iorio, M Pace, P Con, A Gatta
Clinica Medica 5, University of Padua, Padua, Italy F Bortolotti P Con

A Gatta

Hepatology Unit, Hospital Infantil La Paz, Madrid, Spain P Jara

L Hierro

Pediatric Clinic, University of Turin, Turin, Italy

C Barbera

M Pace

Pediatric Hepatology, King's College Hospital, London, UK G V Gregorio G Mieli Vergani

Clinica Pediatrica University of Naples, Italy

A Vegnente

$\mathrm{R}$ Iorio

Department of Pediatrics, University of Padua, Italy

L Zancan

Divisione di Malattie Infettive, Ospedale, Padua, Italy

C Crivellaro

Correspondence to: Dr Flavia Bortolotti, Clinica Medica 5, Policlinico, Via Giustiniani 2, 35100 Padova, Italy

Accepted for publication 25 November 1999

\begin{abstract}
Backgroundlaims-The purpose of this study was to better define the long term prognosis of infection and disease in children with chronic hepatitis $B$ treated with interferon (IFN) alpha.

Patients - A total of 107 children with chronic hepatitis $B$ who received IFN alpha for three or six months in two clinical trials were followed for a mean period of 69 (17) months. Response to treatment was defined as loss of hepatitis $B$ e antigen (HBeAg) within 12 months after stopping treatment. A control group of 59 patients was also followed for a shorter mean time (46 (19) months).

Results-Sixteen (15\%) treated children responded during therapy and $18(17 \%)$ during post-treatment follow up; 31 (29\%) non-responders lost $\mathrm{HBeAg}$ during subsequent years. High pretreatment levels of transaminases and a greater histological activity index were predictors of response. Kaplan-Meier estimates of cumulative $\mathrm{HBeAg}$ clearance rates at five years were similar between treated patients $(60 \%)$ and controls (65\%). After HBeAg clearance, all cases lost hepatitis B virus DNA and $94 \%$ had normal transaminase levels. Loss of hepatitis $B$ surface antigen (HBsAg) occurred in four $(25 \%)$ patients who responded during treatment but in none of the other treated or untreated patients.

Conclusions-After five years' observation, the proportion of treated children with sustained HBeAg clearance comprised an equal number of responders and non-responders and did not differ from that observed in untreated controls, suggesting that IFN simply accelerated a spontaneous event. However, IFN significantly improved the rate of HBsAg loss in cases with more prominent disease activity who were early responders, and may be particularly useful in this type of patient. (Gut 2000;46:715-718)
\end{abstract}

Keywords: hepatitis B; interferon; children; hepatitis B virus

In endemic areas, infection with hepatitis $\mathrm{B}$ virus (HBV) is a major cause of liver disease in childhood. Clinically, chronic hepatitis B is a mild disease in both infants and children and cirrhosis is rare; however, viral replication and liver damage may persist for several years. ${ }^{12}$ In contrast, epidemiological studies in adults with chronic HBV infection have shown that cirrhosis and hepatocellular carcinoma are frequently found in patients infected early in life. ${ }^{3}$ In an attempt to shorten the replicative phase of the illness and thus induce remission of the associated liver disease, interferon (IFN) treatment has been used experimentally in children with chronic hepatitis B in recent years, although only few studies included large numbers of patients. ${ }^{4-8}$

IFN was found to hasten significantly seroconversion of hepatitis $\mathrm{B}$ e antigen ( $\mathrm{HBeAg}$ ) to $\mathrm{HBeAg}$ antibody (anti-HBe) and HBV DNA clearance, inducing a two- to threefold increase in the spontaneous seroconversion rate over an 18 month period. ${ }^{5-8}$ The long term outcome of infection and disease in children who respond to treatment compared with non-responders and to untreated children is still unknown. Recent reports in adults suggest that treatment improves prognosis in terms of survival, progression to cirrhosis, and clearance of HBsAg. ${ }^{9-11}$ Hence the purpose of this study was to investigate the long term outcome of chronic hepatitis B in 107 treated children previously included in published controlled trials.

\section{Patients and methods}

THERAPEUTIC TRIALS

Patients included in this study were children with chronic hepatitis $\mathrm{B}$ randomised to receive IFN or no treatment in two European clinical research protocols. ${ }^{67}$ Eligibility criteria included: (i) age $2-14$ years (first trial) or 2-16 years (second trial); (ii) $\mathrm{HBsAg}, \mathrm{HBeAg}$, and HBV DNA positivity in serum for six months or more; (iii) elevation of serum aspartate aminotransferase (AST) or alanine aminotransferase (ALT) levels for six months or more; (iv) histological evidence of chronic hepatitis without cirrhosis in a liver biopsy obtained within 12 months; and (v) exclusion of coinfection with hepatitis delta or acquired immunodeficiency virus, and of other potential causes of liver damage such as metabolic or autoimmune disorders.

Response to IFN was defined as sustained HBeAg clearance within 12 months after stopping treatment.

The first clinical trial was conducted between 1989 and 1992 in five Italian institutions and included 77 patients randomly assigned to

Abbreviations used in this paper: $\mathrm{HBV}$, hepatitis $\mathrm{B}$ virus; $\mathrm{HBeAg}$, hepatitis $\mathrm{B}$ e antigen; $\mathrm{HBsAg}$, hepatitis B surface antigen; IFN, interferon; ALT, alanine aminotransferase; anti-HBe, antibody to $\mathrm{HBeAg}$; HAI, histological activity index. 
Table 1 Characteristics of patients at presentation

\begin{tabular}{llll}
\hline & First trial $(n=33)$ & Second trial $(n=74)$ & Controls $(n=59)$ \\
\hline Sex $(\mathrm{M} / \mathrm{F})$ & $21 / 12$ & $46 / 28$ & $30 / 29$ \\
Age (years) (mean (SD)) & $8.1(2.9)$ & $9.1(3.5)$ & $8.51(2.5)$ \\
Source of infection & & 0 & 0 \\
$\quad$ Transfusion & 0 & $16(22 \%)$ & $13(22 \%)$ \\
HBsAg+ mother & $5(15 \%)$ & $43(58 \%)$ & $34(58 \%)$ \\
Household contact & $20(61 \%)$ & $15(20 \%)$ & $12(20 \%)$ \\
$\quad$ Other or unknown & $8(24 \%)$ & $3.4(3.6)$ & $2.2(2.0)$ \\
ALT (IU/l)† (mean (SD)) & $2.6(1.5)$ & Minimal hepatitis, $18(24 \%)$ & Minimal hepatitis, $5(8 \%)$ \\
Histological diagnosis $\neq$ & Minimal hepatitis, 1 (3\%) & Chronic hepatitis of mild to \\
& Chronic persistent or chronic & Chronic hepatitis of mild to & moderate activity (or chronic \\
& active hepatitis (mild to & moderate activity, 56 (76\%) & mersistent+active hepatitis), 54 \\
& moderate, 32 (97\%)) & & (92\%) \\
\hline
\end{tabular}

${ }^{\star}$ Known to be HBsAg positive at delivery; ${ }^{\star \star}$ including the mother with unknown virological status at delivery.

†Times upper normal value.

$\ddagger$ For histological classification see text.

receive IFN alpha 2a (Roferon, Roche) at a dose of $3 \mathrm{MU} / \mathrm{m}^{2}$ three times a week for six months (19 cases) or $7.5 \mathrm{MU} / \mathrm{m}^{2}$ three times a week for six months (20 cases), or no treatment (37 cases). Only 61 children recruited in Turin and Padua, however, were considered for follow up.

The second trial was conducted in Italy, Spain, and Great Britain between 1989 and 1993 and included 95 patients randomly assigned to receive lymphoblastoid IFN (Wellferon) alone at a dose of $5 \mathrm{MU} / \mathrm{m}^{2}$ for 12 weeks (34 cases) or IFN at the same dose but preceded by a four week course of prednisolone (30 cases), or no treatment (31 cases). Thirteen additional patients treated with IFN alone (six cases) or with IFN and steroids (four cases), but excluded from the protocol because of an error in randomisation, were included in the study.

Overall, in the two trials there were 59 untreated control cases (only those from Turin and Padua were included in the first trial).

FOLLOW UP OF PATIENTS

After termination of the therapeutic trials all treated and untreated children were invited for follow up visits every $6-12$ months in the outpatient clinic.

Periodic evaluations included: clinical history and physical examination, AST, ALT and serum protein testing, and serological $\mathrm{HBV}$ markers (HBsAg and antibody, $\mathrm{HBeAg}$ and anti-HBe, HBV DNA). Anti-delta antibody was investigated when required. A serum sample was collected at each visit and stored at $-20^{\circ} \mathrm{C}$.

METHODS

HBsAg and antibody to HBs, HBeAg and anti$\mathrm{HBe}$, and anti-delta antibody were measured using commercial assays (Abbott Laboratories, Chicago, Illinois, USA). HBV DNA was inves-

Table 2 Number and percentage of patients who cleared HBeAg at different times during the two therapeutic trials

\begin{tabular}{llll}
\hline Time & $\begin{array}{l}\text { First trial treated } \\
\text { cases }(n=33)\end{array}$ & $\begin{array}{l}\text { Second trial treated } \\
\text { cases }(n=74)\end{array}$ & $\begin{array}{l}\text { First and second trial } \\
\text { controls }(n=59)\end{array}$ \\
\hline End of treatment & $6(18 \%)^{\star}$ & $10(13 \%)^{\star \star}$ & $2(3 \%)$ \\
Six months after stopping treatment & $9(27 \%) \dagger$ & $23(31 \%) \dagger \dagger$ & $5(8 \%)$ \\
12 months after stopping treatment & $9(27 \%) \ddagger$ & $25(34 \%) \neq \ddagger$ & $8(13.5 \%)$ \\
\hline${ }^{\star} \mathrm{p}<0.05,{ }^{\star \star} \mathrm{p}=0.06 v$ controls. & & & \\
$\dagger \mathrm{p}<0.05, \dagger+\mathrm{p}<0.01 v$ controls. & & & \\
$\ddagger \mathrm{p}=\mathrm{ns}, \neq \dagger \mathrm{p}<0.05 v$ controls. & & &
\end{tabular}

tigated by dot blot hybridisation using a quantitative method in the second $\operatorname{trial}^{7}$ and a semiquantitative method in the first. ${ }^{6}$

Liver histology obtained at the beginning of the second trial was classified using a Knodell score modified by Desmet and colleagues. ${ }^{12} \mathrm{~A}$ previous histological classification (De Groote et al) was used in the first trial. ${ }^{13}$

Statistical analysis of the data was performed using the $\chi^{2}$ test, Fisher's exact test, the Student's $t$ test and Wilcoxon's test. The Kaplan-Meier method was used to estimate time to $\mathrm{HBeAg}$ clearance.

\section{Results}

Baseline features in treated and untreated children are reported in table 1: 10 were of Asian origin, three were Black and the remainder were Caucasian.

RESPONSE TO IFN

Table 2 summarises the clearance rates of $\mathrm{HBeAg}$ in treated and untreated children during the trials. Of 34 responders to IFN, 16 cleared $\mathrm{HBeAg}$ during treatment and 18 during the 12 month post-treatment follow up. There was no significant difference in the rate of responders between placebo and steroid pretreated children included in the second trial. Baseline features in responders and nonresponders are shown in table 3. ALT levels were significantly higher in responders and in the second trial the histological activity index (HAI) was greater and HBV DNA levels lower than in responders. There was a trend for higher doses of IFN to be more effective but the group of children treated with low doses was too small for statistical analysis.

CLINICAL EVENTS DURING FOLLOW UP

Overall 107 treated cases were followed up for 18-86 months (mean 69 (17) months) after starting treatment, including 34 responders and 73 non-responders (three non-responders were excluded from the study because they were retreated during follow up). Duration of follow-up in responders and non-responders was similar in the two trials (mean $69.8 v 70.1$ months).

In untreated children, eight were lost to follow up shortly after the end of the trial and 14 received IFN with other treatment schedules at 
Table 3 Baseline features in responders and non-responders

\begin{tabular}{lll}
\hline & Responders $(n=34)$ & Non-responders $(n=73)$ \\
\hline Sex (M/F) & $23 / 11$ & $44 / 29$ \\
Age (years) (mean (SD)) & $8.9(3.5)$ & $8.8(3.4)$ \\
Duration of infection (years) & $2.5(1.9)$ & $3.2(2.1)$ \\
Source of infection & $11(32 \%)$ & $24(33 \%)$ \\
$\quad$ HbsAg+ mother & $11(32 \%)$ & $35(47 \%)$ \\
Household contact & $12(36 \%)$ & $14(20 \%)$ \\
Other or unknown & $4.3(3.0)^{\star}$ & $2.3(2.1)^{\star}$ \\
ALT (mean (SD)) $\ddagger$ & $4.0(1.5)^{\star \star}$ & $3.1(1.3)^{\star \star}$ \\
Mean HAI (mean (SD)) & $8 / 24(33 \%) \dagger$ & $7 / 50(14 \%) \dagger$ \\
HBV DNA <1000 pg/ml & &
\end{tabular}

$\ddagger$ Times upper normal value.

§HAI, histological activity index (for scoring system see text).

${ }^{\star} \mathrm{p}<0.05 ;{ }^{\star \star} \mathrm{p}<0.02$, calculated only for children in the second trial; $t \mathrm{p}=0.07$, calculated only for children in the second trial. months after stopping IFN. None of the other treated or untreated children lost HBsAg during follow up $(\mathrm{p}<0.05 v$ early responders $)$.

BIOCHEMICAL EVENTS DURING FOLLOW UP

Among the responders to treatment, all but two children had normal ALT levels after anti-HBe seroconversion. Two patients continued to have abnormal ALT levels throughout follow up, with peaks of 9.1 and 2.7 times normal, respectively, in the absence of HBV DNA or any other known cause of liver damage. Occasional mild ALT abnormalities with maximum levels of 2.5 times normal were observed in four other cases during follow up, in the absence of detectable viraemia, HDV superinfection, infection with other hepatotropic viruses, or metabolic or autoimmune disorders.

Of the 31 non-responders who cleared HBeAg during follow up, 29 had normal ALT levels after anti-HBe seroconversion. Two patients continued to have abnormal ALT levels, ranging from 1.5 to 3 times normal, during follow up in the absence of viraemia or other known causes of liver damage; occasional ALT elevations of 2-2.5 times normal were observed during follow up in one case, while reactivation, defined as reappearance of HBV DNA and ALT abnormalities after anti-HBe seroconversion, occurred in a 10 year old male 36 months after seroconversion. This patient became jaundiced, developed 100-fold increase in ALT and remained anti-HBe positive. When the ALT flare subsided, HBV DNA again disappeared from serum. No other cause of acute liver damage was identified during reactivation. Of the 42 non-responders who maintained $\mathrm{HBeAg}$ positivity, none had normal ALT levels for any length of time.

Among untreated patients, 28 of 29 who lost HBeAg also had normal ALT levels: one patient had mild ALT abnormalities during follow up in the absence of viraemia and other known causes of liver damage. None of the untreated children who maintained $\mathrm{HBeAg}$ seropositivity had normal ALT levels for any length of time.

VIROLOGICAL EVENTS DURING FOLLOW UP Among the treated children, the 34 responders maintained HBeAg seronegativity while 31 (29\%) of 73 non-responders lost HBeAg. Overall, loss of HBeAg was not influenced by steroid pretreatment.

Also, 29 (49\%) untreated children cleared $\mathrm{HBeAg}$ during follow up. Figure 1 shows the cumulative probability of $\mathrm{HBeAg}$ clearance over the years in treated and untreated children: the final clearance rate did not differ significantly between groups. Loss of $\mathrm{HBeAg}$ was invariably associated with anti-HBe seroconversion. Both non-responders and untreated children who remained $\mathrm{HBeAg}$ positive during follow up tended to maintain HBV DNA positivity throughout the observation: only three cases had lost viraemia at the last visit.

HBs Ag clearance occurred in only four (25\%) of 16 responders who cleared $\mathrm{HBeAg}$ during treatment and lost HBsAg 6-60 months later. One additional early responder cleared HBsAg during treatment but was HBsAg positive four

\section{Discussion}

Our study has described the outcome of chronic hepatitis B in 107 children treated with IFN in two clinical trials. Although the treatment schedules were different we pooled the patients in the light of the following observations: there was no clear evidence in the literature that different types of IFN influence seem to be more effective than low doses, the number of children treated with a low dose ( 3 $\mathrm{MU} / \mathrm{m}^{2}$ ) in this study was small and thus was unlikely to interfere significantly with the outcome of infection and disease; in the second trial, outcome was similar in children pretreated with steroids compared with those who received placebo. Overall, the response to treatment, defined as $\mathrm{HBeAg}$ clearance within 12 months after stopping IFN, was observed in $32 \%$ of cases in this study, a value similar to that recently reported by Sokal and colleagues ${ }^{8}$ in a multicentre controlled study of 72 children the response rate; although high doses of IFN 
treated with a unique schedule. In both studies the rate of $\mathrm{HBeAg}$ clearance was significantly greater in treated than in untreated children 6-12 months after stopping treatment.

Different predictors of response to treatment have been indicated in published studies in adults and children. ${ }^{5-7} 10111415$ Our data confirm that higher baseline ALT activity, greater HAI, and lower HBV DNA levels are significantly associated with response to treatment.

During an average follow up of 69 months, all responders remained $\mathrm{HBeAg}$ and $\mathrm{HBV}$ DNA negative but approximately $50 \%$ of nonresponders also cleared $\mathrm{HBeAg}$, supporting the concept that IFN accelerates the spontaneous course of infection in children with greater disease activity and lower levels of replication. This hypothesis is further strengthened by the similar rates of $\mathrm{HBeAg}$ clearance observed at five years in treated and untreated children. In the latter group, exclusion of $\mathrm{HBeAg}$ positive cases who received treatment could have biased the clearance rate; nevertheless this rate is similar to that described recently in a large cohort of untreated Italian and Spanish children. ${ }^{16}$

Interestingly, the rate of $\mathrm{HBsAg}$ clearance was significantly greater $(25 \%)$ in children who responded promptly to treatment than in late responders, non-responders, or untreated controls. This finding is in keeping with recent reports in adults. ${ }^{911}$ Thus IFN seems to favour loss of HBsAg, particularly if given to patients who are more prone to respond to treatment, such as those with active disease and low levels of virus replication.

During follow up, the majority of treated and untreated children who cleared viraemia and HBeAg had sustained normal levels of ALT. Only in one non-responder was there a bout of reactivation of virus replication and liver disease. Mild or moderate increases in ALT in the absence of detectable viraemia were more frequent in treated children. In these cases low levels of virus replication detectable only by polymerase chain reaction or by selection of yet undetermined $\mathrm{HBV}$ mutants might sustain liver damage. ${ }^{17}$

The benefits of IFN (shortening the replicative phase of the illness and increasing the rate of HBsAg clearance) in a subgroup of children must be weighted against the side effects of treatment. Indeed serious adverse events attributable to IFN seem to be rare in children. ${ }^{8}$ In this study, only one patient experienced a serious complication (autoimmune thrombocytopenia); all other symptoms were transient and disappeared after withdrawal of therapy.

One major issue concerning the efficacy of IFN is whether treatment can modify the natural history of the disease. Recent observations in adults indicate that treatment improves the survival rate and decreases the chance of evolution to cirrhosis. ${ }^{11}{ }^{11}$ These end points could not be evaluated in our series. In fact the results of our 20 year follow up study in untreated children in the Mediterranean area have shown that cirrhosis is a rare and early complication of chronic infection rather than the late stage of ongoing disease, and that hepatocellular carcinoma occurs only in some cirrhotic children. ${ }^{16}$

In conclusion, the results of our study showed that after five years of observation the proportion of treated children with sustained HBeAg clearance comprised an equal number of responders and non-responders, and did not differ from that observed in untreated controls, suggesting that treatment only accelerates a spontaneous event. However, IFN significantly improved the rate of HBsAg loss in cases with more prominent disease activity who were early responders, and could be particularly useful in this type of patient.

We are indebted to Professor C Merkel for help in statistical analysis.

1 Ruiz Moreno M, Camps T, Garcia Aguado J, et al. A serological and histological follow-up of chronic hepatitis B infection. Arch Dis Child 1989;64:1165-9.

2 Bortolotti F, Cadrobbi P, Crivellaro C, et al. Long-term outcome of chronic type B hepatitis in patients who acquire hepatitis B virus infection in childhood. Gastroenterology 1990;99:805-10.

3 Beasley RP. Hepatitis B virus. The major etiology of hepatoBeasley RP. Hepatitis B virus. The major et
cellular carcinoma. Cancer $1988 ; 6: 1942-5$.

4 Lai CL, Lin HJ, Lau HJ, et al. Effect of recombinant alpha 2 IFN with or without prednisone in Chinese HBsAg carrier children. QfM 1991;78:155-63.

5 Ruiz Moreno M, Rua MJ, Molina J, et al. Prospective, randomized, controlled trial of IFN alpha in children with chronic hepatitis B. Hepatology 1991;13:1035-9.

6 Barbera C, Bortolotti F, Crivellaro C, et al. Recombinant interferon $\alpha 2 \mathrm{a}$ hastens the rate of $\mathrm{HBeAg}$ clearance in children with chronic hepatitis B. Hepatology 1994;20:287-90.

7 Gregorio M, Jara P, Hierro L, et al. Lymphoblastoid interferon alpha with or without steroid pretreatment in children with chronic hepatitis B: a multicentre controlled trial. Hepatology 1996;23:700-7.

8 Sokal EM, Conjeevaram H, Roberts EA, et al. Interferon alpha for treatment of pediatric chronic hepatitis B: a randomized controlled multicenter trial. Gastroenterology 1998; 114:988-95.

9 Korenman J, Baker B, Waggoner J, et al. Long-term remission of chronic hepatitis B after alpha-interferon therapy. Ann Intern Med 1991;114:629-34.

10 Niederau C, Heintges T, Lange S, et al. Long-term follow-up of $\mathrm{HBeAg}$ positive patients treated with interferon alfa for chronic hepatitis B. New Engl $f$ Med 1996;334:1422-7.

11 Lau DT, Everhart J, Klainer DE, et al. Long-term follow-up of patients with chronic hepatitis B treated with interferon alfa. Gastroenterolgy 1997;113:1660-7.

12 Desmet VJ, Gerber M, Hoofnagle JH, Manns M, Sheuer PJ. Classification of chronic hepatitis: diagnosis, grading and staging. Hepatology 1994;19:1513-20.

13 De Groote J, Desmet VJ, Gedigk P, et al. A classification of chronic hepatitis. Lancet 1968;2:626-8.

14 Ruiz-Moreno M, Camps T, Jimenez J, et al. Factors predictive of response to interferon therapy in children with chronic hepatitis B. F Hepatol 1995;22:540-4.

15 Lau GKK, Lim W, Lock ASF. Nine years follow-up after interferon (IFN) treatment for chronic hepatitis B (CHB) in Chinese patients. Hepatology 1997;26:259A.

16 Bortolotti F, Jara P, Crivellaro C, et al. Outcome of chronic hepatitis $\mathrm{B}$ in Caucasian children during a 20-year observation period. F Hepatol 1998;29:184-90

17 Bortolotti F, Wirth S, Crivellaro C, et al. Long-term persistence of hepatitis $B$ virus DNA in the serum of children with chronic hepatitis B after hepatitis B e antigen to antibody seroconversion. F Pediatr Gastroenterol Nutr 1996;22:270-4. 